\title{
Adaptation of TDMA Parameters Based on Network Conditions
}

\author{
Bora Karaoglu \\ Dept. of Elect. and Computer Eng. \\ University of Rochester \\ Rochester, NY 14627 \\ Email: karaoglu@ece.rochester.edu
}

\author{
Tolga Numanoglu \\ Dept. of Elect. and Computer Eng. \\ University of Rochester \\ Rochester, NY 14627 \\ Email: numanogl@ece.rochester.edu
}

\author{
Wendi Heinzelman \\ Dept. of Elect. and Computer Eng. \\ University of Rochester \\ Rochester, NY 14627 \\ Email: wheinzel@ece.rochester.edu
}

\begin{abstract}
Soft clustering of the nodes combined with time division multiple access (TDMA) channel access within a cluster has been shown to provide an energy-efficient solution for Mobile Ad-Hoc Networks (MANET). Such channel access schemes use a parameter that is critical in determining network performance: the number of frames per superframe, which determines the amount of spatial reuse possible, similar to the frequency reuse factor in cellular networks. When a smaller number of frames per superframe is used, each frame will consist of a larger number of slots, enabling the frame (i.e., cluster) to support more nodes, but also limiting the choices of frames for clusterheads to select, causing higher co-channel interference and collisions. Conversely, when a larger number of frames per superframe is used, the clusterheads will only be able to grant channel access to a limited number of nodes, which in turn increases the number of dropped packets (i.e., blocked channel access). The optimum value of the number of frames is the one that minimizes the combined effect of both collisions and dropped packets. By analytically determining the effects of dropped packets and collisions, we can find the optimal value for any given scenario. This paper develops a model to determine the optimal TDMA structure under various settings, showing the advantages that can be obtained by adapting protocol parameters as network conditions change.
\end{abstract}

\section{INTRODUCTION}

The MAC protocol is the key element in the protocol stack that determines the ability of a wireless network to meet application requirements, since the MAC protocol has a direct impact on throughput, Quality of Service (QoS), energy dissipation, fairness, stability, and robustness [1], [2].

In particular, coordinated channel access schemes provide support for QoS, reduce energy dissipation and increase throughput for low-to-mid noise levels and for dense networks [3]. MH-TRACE [4] and IEEE 802.15.3 [5] are examples of such coordinated protocols. The IEEE 802.15.3 protocol is specifically designed for high-rate and short range WPAN networks [6]. MH-TRACE is designed for mid-range mediumrate transmissions [4]. Both of those algorithms use a TDMA structure together with soft clustering of the nodes for channel access, as this approach has been shown to provide satisfactory performance in terms of QoS and energy dissipation.

Many of the protocol parameters in cluster-based protocols are set a-priori based on estimates of network conditions

This work was supported in part by the University of Rochester Center for Electronic Imaging Systems and in part by Harris Corporation, RF Communications Division. and based on a specific physical layer. TDMA parameters, which determine the amount of spatial reuse and interference, distribute the available bandwidth among clusters so as to reduce the interference throughout the network. Reducing the interference is a desirable goal since high interference leads to high error rates, decreasing the throughput as studied in [7]. However, reducing the available bandwidth per cluster also decreases the capacity per cluster. The non-uniform distribution and node mobility may increase the local load above the cluster capacity, resulting in dropped packets and decreasing the throughput for real-time traffic [4]. The decision of how to set these parameters should thus be based on this trade-off and would be effected by various conditions such as node density and physical layer parameters.

The relationship between the parameters can only be determined by analytical analysis. Although simulation studies reveal the performance of a protocol for a certain set of conditions, the statistical accuracy of the simulation results is questionable unless repeated extensively. For large and dense networks, this approach requires excessive amounts of processing power. Moreover, results obtained from those studies are only valid for the selected parameters and do not reveal their impact on the performance of the protocol. Therefore, we have developed an analytical model that reflects the relationships between protocol parameters and the overall performance of the protocol under different network conditions for the TDMA-based clustered protocol MH-TRACE. Specifically, we develop a model that relates the TDMA frame parameters (number of slots per frame and number of frames per superframe) and the node density to the expected number of dropped packets and the expected number of collisions. This model enables us to find the set of parameters that maximize overall throughput for MH-TRACE, and, by extension, for TDMA-based clustered protocols such as IEEE 802.15.3.

The organization of this paper is as follows. In section II, a brief summary of MH-TRACE is presented. An analytical model that helps in identifying the performance of $\mathrm{MH}$ TRACE for a given set of parameters is presented in section III. Section IV discusses the validity of the analytical model and protocol parameter decisions based on that model by comparing the analytical results with those found via simulation. Section V concludes the discussion with final comments. 


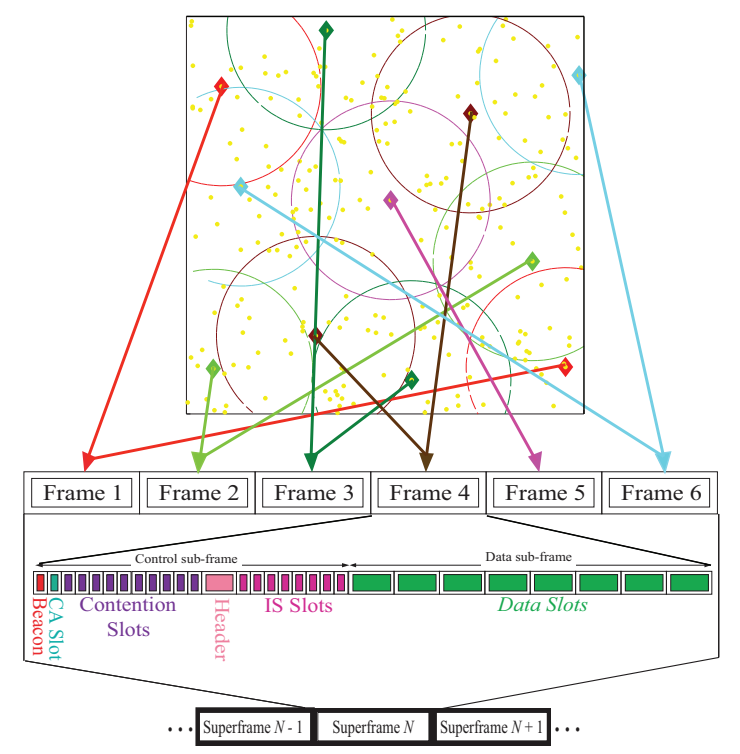

Fig. 1. A snapshot of MH-TRACE clustering and medium access. Diamonds represent selected clusterheads and dots represent the nodes in the network. $\mathrm{CH}$-frame matching together with the contents of each frame is depicted.

\section{BACKGROUND: MH-TRACE}

In order to understand the results of the study, it is important to understand how the MH-TRACE protocol works. This section briefly explains the protocol's crucial mechanisms and aims to give the reader a brief understanding of the protocol. The complete protocol description is available in [4].

In MH-TRACE, time is divided into superframes of equal length, as shown in Fig. 1, where the superframe is repeated in time and further divided into frames. Each cluster operates in one of the frames, and the clusterhead $(\mathrm{CH})$ manages the channel access scheme of that frame. There is a spatial reuse mechanism that allows more than one cluster to operate in the same time frame provided that the interference is low enough.

Each frame in the superframe is further divided into subframes. The control sub-frame is used for management overhead. The $\mathrm{CH}$ selection and maintenance as well as interference minimization algorithms use the $\mathrm{CH}$ announcement and beacon slots of the control sub-frame. The rest of the control sub-frame is devoted to the proper collection of channel access requests and announcement of channel access grants as well as data summarization. The data subframe contains data slots where nodes that are successful in securing channel access transmit their data in the order announced by the $\mathrm{CH}$.

\section{AnAlyticAl AnAlysis}

In TDMA-based clustered protocols such as MH-TRACE, there are two main factors limiting the performance (in terms of throughput); dropped packets and collisions. Thus, our goal is to model the effect of the protocol parameters on both the expected number of dropped packets and the expected number of collisions in order to estimate the throughput.

MH-TRACE is an ideal protocol for supporting real-time traffic, such as voice streaming, where a data stream is divided into several packets of fixed length. In order to maintain continuity of the stream, data slots should be assigned periodically to nodes that have data to send, and this period must be matched to the length of each packet and the data frequency (e.g., voice sampling rate). This provides bounds on some of the TDMA parameters, such as slot size and superframe time, in order to guarantee that the traffic meets its delay bounds.

In MH-TRACE, the nodes within a cluster request channel access from the $\mathrm{CH}$, and the $\mathrm{CH}$ grants the channel access by assigning available data slots to the nodes that request channel access. However, the number of data slots is limited and mainly determined by the length of the frame. If the $\mathrm{CH}$ grants channel access and assigns a data slot to a node, it continues to reserve the same data slot in consecutive superframes as long as the node's transmission continues. Thus, the time interval between the data slots in two consecutive superframes, which is equal to the superframe duration, is fixed and determined by the length of each packet and the sampling rate.

Given that the length of the superframe must be matched to the real-time data frequency, as the number of frames in the superframe $\left(N_{f}\right)$ is increased, each frame must be shorter and thus has fewer data slots. This increases the probability of having no available data slot when a node requests channel access from the $\mathrm{CH}$. The packet must be dropped due to real time traffic requirements. On the other hand, as $N_{f}$ is decreased, spatial reuse is increased which increases capacity per cluster but also increases the interference and collisions. Thus, there is a trade-off in terms of throughput between dropped packets and collisions, and our goal is to model both of these sources of throughput loss in order to analytically examine this trade-off.

For a fixed physical layer and voice model, our model provides an estimate of the expected number of dropped packets and the expected number of collisions based on the values of the following parameters: $N_{f}$, the network area, and the number of nodes in the network. Throughput is estimated based on the number of dropped packets and the expected number of collisions, as described in the following sections.

\section{A. Dropped Packets}

The probability of dropped packets can be calculated as

$$
P_{d p}=\max \left(\frac{\text { Load }- \text { Capacity }}{\text { Load }}, 0\right),
$$

where Load represents the number of nodes that require channel access within a cluster per superframe and Capacity is the total number of data slots available for the cluster (i.e., the number of data slots per frame). Each cluster is capable of supplying channel access only to a limited number of nodes per superframe that is equal to the number of data slots in the frame.

The load in the cluster is mainly determined by three probabilities:

- $p_{s}$ : Probability of a node to be in spurt duration,

- $p_{A}$ : Probability of a node to be in the communication range of a $\mathrm{CH}$. 
- $p_{d}$ : Probability of a node that is in the communication range of a $\mathrm{CH}$ to choose that $\mathrm{CH}$ as its channel access provider.

We assume a voice model where nodes are either in "spurt" or "gap", with voice data being generated in the "spurt" duration and no data being generated in the "gap" duration. Hence, $p_{s}$ accounts for the fact that not all nodes will have data to send at a given time. Furthermore, MH-TRACE allows nodes to access the channel from any $\mathrm{CH}$ within range. Therefore, if one $\mathrm{CH}$ has no data slots available, a node can request channel access from another $\mathrm{CH}$ in range. Hence, $p_{d}$ takes into account the fact that a node's expected load to a cluster is distributed among those $\mathrm{CHs}$ in the communication range of that node.

Given those distributions, the load per cluster per superframe for a given $\mathrm{CH}$ (and its location, $\mathrm{CH}_{l o c}$ ) is a binomial distribution with a success probability of $p_{d n}=p_{s} p_{A} p_{d}$ as given in (2).

$\operatorname{Pr}\left(\right.$ Load $\left.=k \mid C H_{\text {loc }}\right)=\left(\begin{array}{c}N_{\text {nodes }} \\ k\end{array}\right) p_{d n}{ }^{k}\left(1-p_{d n}\right)^{\left(N_{\text {nodes }}-k\right)}$

1) Probability of a node to be in spurt duration $\left(p_{s}\right)$ : The voice model we use incorporates exponentially distributed spurt and gap durations ( $T_{\text {spurt }}$ and $T_{\text {gap }}$, respectively) followed by each other. The probability of finding a node in spurt state can be calculated as given in (3).

$$
\operatorname{Pr}(\text { Node } i \text { in spurt })=\frac{E\left[T_{\text {spurt }}\right]}{E\left[T_{\text {spurt }}+T_{\text {gap }}\right]}=p_{s}
$$

2) Probability of a node to be in the communication range of a $\mathrm{CH}\left(p_{A}\right)$ : Nodes are assumed to be i.i.d. distributed according to a uniform distribution. Thus, the probability of a node to be in any given region is the area of that region divided by the whole simulation area.

$$
\operatorname{Pr}(\text { Node in Region } \mathrm{A})=\frac{\text { Area of Region } \mathrm{A}}{\text { Simulation Area }}=p_{A}
$$

The coverage region of a cluster is a circle centered at the $\mathrm{CH}$ 's location having a radius equal to the maximum reliable communication distance at the selected power level. Part of a CH's coverage region may lie outside the network area depending on the CH's location, $\mathrm{CH}_{l o c}$. The network area can be divided into 3 regions, as in Fig. 2. The coverage area that lies within the simulation area, $\alpha_{1}, \alpha_{2}$, and $\alpha_{3}$ for a $\mathrm{CH}$ in region 1, 2 and 3, respectively, are calculated in [7] and repeated here for reference.

$$
\begin{aligned}
\alpha_{1} & =\pi r^{2}, \\
\alpha_{2} & =\pi r^{2}-2 I\left(d_{0}\right), \\
\alpha_{3} & =\frac{3}{4} \pi r^{2}-I\left(x_{0}\right)-I\left(y_{0}\right)+x_{0} y_{0},
\end{aligned}
$$

where $x_{0}$ and $y_{0}$ are the distances to the closest vertical (for $x_{0}$ ) and horizontal (for $y_{0}$ ) limits of the network area, $d_{0}=$ $\min \left(x_{0}, y_{0}\right)$ and the function $I(x)$ is as given in

$$
I(x)=\frac{\pi}{4} r^{2}-\frac{x}{2} \sqrt{r^{2}-x^{2}}-\frac{r^{2}}{2} \arcsin (x / r) .
$$

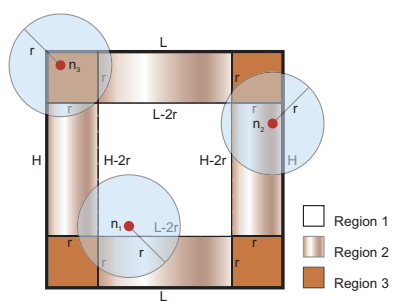

Fig. 2. Partitioning of the simulation region. Part of the coverage region lies outside the network region for $\mathrm{CHs}$ located in regions 2 and 3.

Replacing Region $A$ with the appropriate term from (5), leads to desired $p_{A}$ for a given $\mathrm{CH}$.

3) Probability of a node to be in the communication range of a $\mathrm{CH}\left(p_{d}\right)$ : The entire simulation area will be fully covered for sufficiently large node densities. After that point, variations in the node density do not alter the expected number of cluster head nodes in the network and their positions. Thus, the number of $\mathrm{CHs}$ in the communication range of each node is independent of node density for sufficiently large node densities. Simulations indicate that $35 \%$ of the nodes are in the communication range of only one $\mathrm{CH}$ whereas $48 \%$ and $17 \%$ of the nodes are in the communication range of two and three $\mathrm{CHs}$, respectively for a communication range of $250 \mathrm{~m}$. Nodes can select their channel access provider, uniformly among the $\mathrm{CHs}$ that are in their communication range. Using the aforementioned values $p_{d}$ can be calculated as 0.6467 .

Substituting (5a)-(5c) into (4) and (2), $p_{A}$ and the statistical distribution of load can be obtained for a given $\mathrm{CH}$. Taking a weighted average, using the probability of CH's locations as the weights, gives the distribution of load for a generic $\mathrm{CH}$ as

$$
\operatorname{Pr}(\text { Load }=k)=\iint_{S} \operatorname{Pr}\left(\text { Load }=k \mid C H_{l o c}\right) \operatorname{Pr}\left(C H_{l o c}\right) d S,
$$

where $S$ is the simulation area and $\mathrm{CH}_{l o c}$ is a CH's location. The integral in (7) can be taken numerically for all $k$ values.

4) Effect of dropped packets on throughput: Using the distributions of Load, the distribution of $P_{d p}$ is calculated by (1). If a dropped packet was transmitted successfully, that packet would have been received by all the nodes that are in the transmission range of the transmitting node. Thus, each dropped packet decreases the throughput by the number of neighboring nodes. The total decrease in throughput per superframe due to dropped packets, $f_{d r o p}$, is another random variable and is related to $P_{d p}$ by

$$
f_{\text {drop }}=P_{d p} \times\left(P_{s} \times N_{\text {nodes }}\right) \times E\left[N_{\text {neighbors }}\right] .
$$

The expected number of neighbors, $E\left[N_{\text {neighbors }}\right]$, can be calculated as

$$
E\left[N_{\text {neighbors }}\right]=p_{a} \times N_{\text {nodes }},
$$

where $p_{a}$, the probability of a node to be in the communication range of another node, is calculated in a manner similar to the calculation of $p_{A}$. 


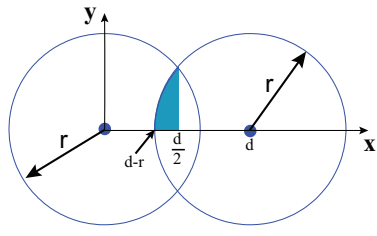

(a)

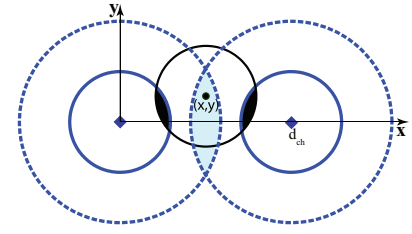

(b)
Fig. 4. (a) Intersection region for two circles with radius $r$ separated by $d$. (b) A typical alignment of co-frame clusters together with their vulnerable region (lightly shaded region) and a node in vulnerable region represented by the disk. The dark shaded region is the region of member nodes whose packets could collide at the node represented by the disk.

\section{B. Collisions}

CHs manage the channel access within the cluster so as to prevent simultaneous transmissions of the nodes in the cluster. Hence, collisions within the cluster are completely eliminated. Clusters operating in separate frames access the channel in distinct time instances, and hence collisions among nodes in these clusters are also not possible. The only remaining source of collisions is co-frame clusters.

Our approach for calculating the number of collisions per superframe includes two steps. The first step is to relate the number of frames per superframe $\left(N_{f}\right)$ to the co-frame $\mathrm{CH}$ separation, and the second step is to relate the co-frame $\mathrm{CH}$ separation to the number of collisions per superframe.

1) Relation between $N_{f}$ and co-frame $C H$ separation: The number of collisions increases as the separation between coframe $\mathrm{CHs}$ decreases. Due to the $\mathrm{CH}$ creation mechanism, the minimum separation between two $\mathrm{CHs}$ is equal to the communication distance, $r$, in the worst case scenario. Under maximum packing conditions, a third $\mathrm{CH}$ is also separated by $r$ from both of the previous $\mathrm{CHs}$ as in Fig. 3. The lines connecting the $3 \mathrm{CHs}$ forms an equilateral triangle. At most $6 \mathrm{CHs}$ can be located around the first $\mathrm{CH}$ as in Fig. 3. Considering an imaginary cell boundary passing perpendicular to the lines connecting the neighboring $\mathrm{CHs}$, a hexagonal cellular structure can be constructed in the form of the standard structure used in cellular systems [8]. The labeling structure that is used in cellular systems is applicable for the clustering structure under concern. Distinct labels correspond to clusters operating in distinct frames in the superframe structure. The separation between co-labeled cells and hence co-frame $\mathrm{CHs}$ is given as

$$
d_{c h}=r \times \sqrt{N_{f}} .
$$

We use this separation as a basis to calculate the collisions, although a regular structure that maximizes the distance between co-frame $\mathrm{CHs}$ exists only for a certain set of $N_{f}$ values.

2) Relation between $\mathrm{CH}$ separation and number of collisions: The first step in determining the relation between the number of collisions and the $\mathrm{CH}$ separation, $d_{c h}$, is to find a function, $f(d, r)$, that calculates the intersection area of two circles having radii $r$, and whose center points are separated by $d$. This function will be used frequently later on. Fig. 4a shows two such circles. From symmetry, the total intersection area is four times the shaded area and can be calculated as

$$
\begin{aligned}
f(d, r) & =4 \int_{d-r}^{\frac{d}{2}} \int_{0}^{\sqrt{r^{2}-(x-d)^{2}}} d y d x \\
& =\left.4 r^{2}\left(\frac{\sin (2 \theta)}{4}+\frac{\theta}{2}\right)\right|_{\theta=-\frac{\pi}{2}} ^{\theta=-\arcsin \left(\frac{d}{2 r}\right)} .
\end{aligned}
$$

Nodes vulnerable to collisions should be located at most 2 times the communication radius, $r$, from a $\mathrm{CH}$. Furthermore, those nodes should also be located within 2 times the communication radius of a co-frame $\mathrm{CH}$. Thus, the vulnerable region for two co-frame $\mathrm{CHs}$ is the area of intersection of two circles with radii $2 r$ and centers located at the co-frame $\mathrm{CH}$ positions. The lightly shaded area in Fig. $4 \mathrm{~b}$ depicts the vulnerable region for two CHs represented by two diamonds. The nodes in the vulnerable region are called vulnerable nodes. As the co-frame $\mathrm{CH}$ separation is decreased, the vulnerable region and hence the number of vulnerable nodes increases.

The nodes whose packets may collide at a node in the vulnerable region should be in the communication range of that node. In Fig. $4 \mathrm{~b}$, the little disk located at $(x, y)$ represents an arbitrarily selected node among the vulnerable nodes. Packets transmitted by the nodes in the dark shaded regions will collide at the selected node when these packets are scheduled at the same data slot. As the co-frame $\mathrm{CH}$ separation is decreased, the area of the dark shaded regions and hence the number of nodes in those regions increases, which in turn leads to an increased probability of collision at the selected node.

The number of nodes whose packets may collide at a selected node in the vulnerable region, $N_{\text {nodes } 2 c o l l}$, is a function of the relative position of the selected node with respect to the $\mathrm{CH}$ locations and the distance between the CHs. The sum of $N_{\text {nodes2coll }}$ for all the nodes in the vulnerable area leads to $T N_{\text {nodes } 2 \text { coll }}$, which is a function of $\mathrm{CH}$ separation.

$N_{\text {nodes } 2 \text { coll }}$, for the disk located at $(x, y)$ in Fig. $4 \mathrm{~b}$, is the sum of the number of nodes in the dark shaded regions and can be calculated by multiplying the node density with the area of these regions as in (12) since nodes are i.i.d. and uniformly distributed.

$$
\begin{aligned}
& N_{\text {nodes } 2 \text { coll }}=d_{n} \times \\
& \quad\left[f\left(\sqrt{x^{2}+y^{2}}, r\right)+f\left(\sqrt{\left(d_{c h}-x\right)^{2}+y^{2}}, r\right)\right],
\end{aligned}
$$

where $d_{n}$ is the node density and $d_{c h}$ is the $\mathrm{CH}$ separation.

Integrating (12) for all nodes in the vulnerable region, $V$, (lightly shaded area in Fig. 4b) yields

$$
\begin{aligned}
& T d_{n}^{2} \iint_{V}\left[f\left(\sqrt{x^{2}+y^{2}}, r\right)+f\left(\sqrt{\left(d_{c h}-x\right)^{2}+y^{2}}, r\right)\right] d x d y .
\end{aligned}
$$

Assuming the $\mathrm{CHs}$ are well separated (making the region of integration small), (13) can be approximated by

$$
T N_{\text {nodes } 2 \text { coll }}=d_{n}^{2} \times f\left(d_{c h}, 2 r\right) \times\left[2 \times f\left(\frac{d_{c h}}{2}, r\right)\right] .
$$


TABLE I

SUPERFRAME PARAMETERS

\begin{tabular}{|c|c|c|c|}
\hline $\begin{array}{c}\text { Number of Frames } \\
N_{f}\end{array}$ & $\begin{array}{c}\text { Number of Data } \\
\text { Slots, } N_{d}\end{array}$ & $\begin{array}{c}\text { Number of Contention } \\
\text { Slots, } N_{c}\end{array}$ & $\begin{array}{c}\text { Superframe } \\
\text { Time } T_{s f}\end{array}$ \\
\hline 4 & 12 & 15 & 24,976 \\
\hline 5 & 10 & 6 & 25,060 \\
\hline 6 & 8 & 9 & 24,984 \\
\hline 7 & 7 & 6 & 25,172 \\
\hline 8 & 6 & 6 & 24,992 \\
\hline
\end{tabular}

The exact relationship between $T N_{\text {nodes } 2 \text { coll }}$ and the number of collisions per superframe, $f_{\text {coll }}$, is complex in nature. We use a first order simplification, and assume that they are linearly related. Under this assumption, the number of collisions can be obtained by scaling $T N_{\text {nodes } 2 \text { coll }}$ with a constant, $C$, as in (15). The constant is independent of $N_{\text {nodes }}$ and $N_{f}$ since those effects are already taken into account in $T N_{\text {nodes } 2 \text { coll }}$ through density and co-frame $\mathrm{CH}$ separation. The constant can be determined by comparing the analytical results with simulation results for sparse networks where the simulation is possible. Using the same constant, $f_{\text {coll }}$ can be determined for any node density and any $N_{f}$ value.

$$
f_{\text {coll }}=C \times T N_{\text {nodes } 2 \text { coll }}
$$

\section{Proof OF CONCEPT}

The aim of the analytical study is to create a model that will enable us to examine the performance of the MH-TRACE protocol for any given values of $N_{f}$, network size and number of nodes. Using this model, for example, we can determine a "good" $N_{f}$ value that maximizes the throughput in the system for any given node density.

The analytical model given above is a first order approximation for a complex system. Thus, the approximation errors may effect the decision about the optimal $N_{f}$ value and may lead to suboptimal solutions. In this section we evaluate the performance of our analytical model in determining the optimal $N_{f}$ value.

\section{A. Simulation Environment}

For comparison purposes, we conduct ns-2 simulations of MH-TRACE under different network scenarios and for various $N_{f}$ values. Voice communications is simulated with "spurts" and "gaps" whose lengths are exponentially distributed and statistically independent with mean durations of $1.0 \mathrm{~s}$ and $1.35 \mathrm{~s}$, respectively[9][10]. The source application is the conversational voice, coded at $32 \mathrm{Kbps} .100$ byte long packets are generated from that application for all nodes. The channel rate is set to $2 \mathrm{Mbps}$. Given these values, in order to maintain data packet timing, the superframe time is adjusted to be as close as possible to $25 \mathrm{~ms}$, which is the voice packet generation period. Table I shows details for each value of $N_{f}$.

Beacon, CA, contention, and IS packets are all 4 bytes long. The header packet has a variable length of $\left(4+2 N_{d}\right)$ bytes depending on the number of data slots. There is a 4 byte header and 100 byte data packet associated with each data slot. Details of the various header packet lengths can be found in [4].

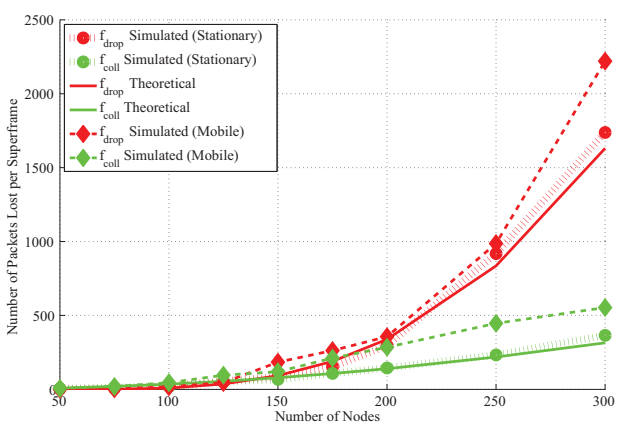

(a) $N_{f}=6$

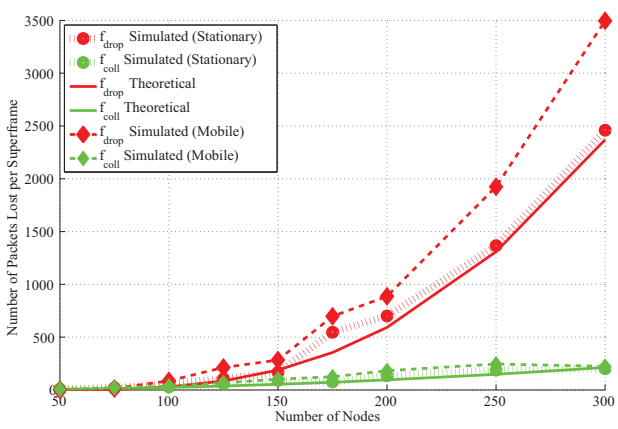

(b) $N_{f}=8$

Fig. 5. Packet loss functions for dropped packets and collisions for both stationary and mobile nodes.

The random way-point mobility model [11][12] is used in mobile scenarios where the node speeds are chosen from a uniform random distribution between $0.0 \mathrm{~m} / \mathrm{s}$ and $5.0 \mathrm{~m} / \mathrm{s}$ with zero pause time. The energy model discussed in [13] and the default propagation model that is available in ns-2 [14] are used. We used a constant transmit power that results in a constant receiving range of $250 \mathrm{~m}$. All simulations are run in an area of $1 \mathrm{~km} \times 1 \mathrm{~km}$ for 100 seconds. The simulations are repeated with the same parameters 5 times. All results are based on sample average of those 5 iterations.

\section{B. Results and Comparison}

The loss in throughput due to collisions and dropped packets are depicted in Fig. 5 for $N_{f}$ equal to 6 and 8, respectively. For stationary case, the analytical estimations that are obtained with the method described in the previous section tightly follow the simulation results for all node densities and all $N_{f}$ values, verifying the model. The simulation results for mobile case follows the same trend as the theoretical results but are not as close as the stationary case. The first reason for this is that the random way-point model results in a non-uniform node distribution by bringing the nodes together[11][12]. The assumption of uniform node density used throughout the theoretical analysis fails as time evolves under the random way-point model. The second reason is that mobility causes $\mathrm{CHs}$ that come near each other to resign and let other nodes form new clusters. During the $\mathrm{CH}$ formation process, the local capacity around resigned $\mathrm{CHs}$ decreases, which in turn leads to a higher number of dropped packets.

Another observation is that as $N_{f}$ is increased, the collisions decrease, whereas the dropped packets increase. Increasing 
$N_{f}$ increases the separation between co-frame clusters which in turn decreases collisions. On the other hand, since the superframe length is fixed, increasing $N_{f}$ decreases the frame length and hence the number of data slots per frame and the capacity per $\mathrm{CH}$. Thus, there is a higher number of dropped packets as $N_{f}$ is increased.

Theoretically, the maximum throughput should be realizable at the $N_{f}$ value that minimizes the combined effects of collisions and dropped packets. Thus, the optimal $N_{f}$ parameter of the analytical model is the value that minimizes the analytical estimate of collisions and dropped packets for a given node density. In order to determine the applicability of using the analytical model for selecting the optimal $N_{f}$ value, we measure the simulated throughput for all $N_{f}$ values as a percentage of the maximum realizable throughput at a given node density. Fig. 6 depicts the performance of the decisions obtained through the analytical estimations together with the worst decision for $N_{f} \in\{4,5,6,7,8\}$ for all node densities and for both the mobile and stationary cases. All decisions obtained through analytical analysis lie within $4 \%$ and $9 \%$ of the maximum realizable throughput for the stationary and mobile cases, respectively. Analytical analysis is successful in determining the optimal $N_{f}$ value for dense networks, in both the mobile and stationary cases, although in the mobile case, individual estimations for dropped packets and collisions obtained from the analytical analysis are not as close to the simulated values as they are in the stationary case.

For low node densities, the demand for channel access is fairly low and the capacity is sufficient. The dropped packets are merely the results of non uniformities in node distribution. Thus, increasing capacity per cluster has relatively less impact on throughput. Also the impact of collisions on throughput is less for sparse networks since collisions are proportional to the square of the node density. Hence, the decision on which $N_{f}$ value to use is relatively less important for sparse networks as can be observed in Fig. 6 by the decreasing performance difference between the worst and best decisions as node density is decreased. On the other hand, the decision about which $N_{f}$ value to use is quite important for dense networks. For 300 node stationary and mobile networks, choosing the worst $N_{f}$ value achieves only $65 \%$ and $64 \%$ of the optimum throughput, respectively. Thus, appropriate setting of the $N_{f}$ parameter is extremely important, and the analytical model provided in this paper can be used to optimally set this value for arbitrary networks.

\section{CONCLUSION}

The analysis made in this paper reveals the importance of proper selection of TDMA parameters according to network conditions. It has been shown that improper selection of these parameters may result in serious throughput loses (up to $35 \%$ for stationary and $36 \%$ for mobile scenarios) in dense networks, while the decision has smaller impact for sparse networks. The primary determining factor in the decision is the node density. Other factors such as transmitting power and noise levels as well as mobility patterns also have an impact

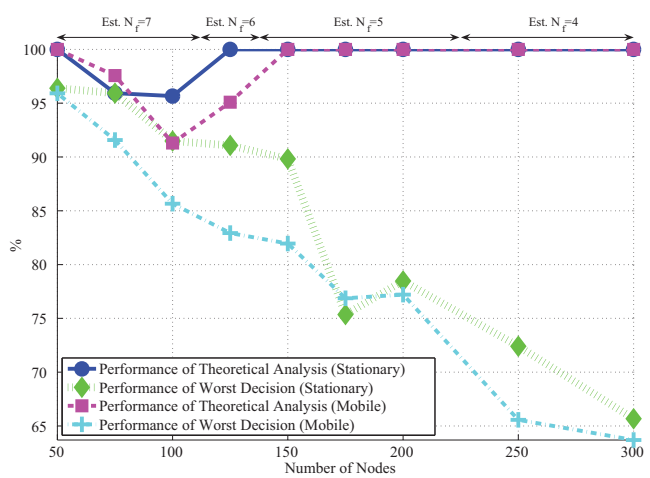

Fig. 6. Performance of the $N_{f}$ decision of the analytical analysis together with the worst decision as a percentage of the best decision for all node densities. Selected $N_{f}$ values through analytical analysis is displayed on the top of the graph.

but they are out of the scope of this paper and are the subjects for future work.

The analytical analysis presented in this paper not only makes the analysis possible for very dense networks where the simulation of the network is impractical but also reveals the impact of parameters on system performance. It should be emphasized that, although the analytical model developed in this paper is specifically designed for MH-TRACE, it can be easily adapted to any protocol where the bandwidth is shared among clusters.

\section{REFERENCES}

[1] A. Chandra, V. Gummalla, and J. O. Limb. Wireless medium access control protocols. IEEE Communications Surveys and Tutorials, 3:2$15,2000$.

[2] P. Mohapatra, J. Li, and C. Gui. Qos in mobile ad hoc networks. IEEE Wireless Communications Magazine, 10:44-52, 2003.

[3] T. Numanoglu, B. Tavli, and W. Heinzelman. An analysis of coordinated and non-coordinated medium access control protocols under channel noise. Military Communications Conference, 2005. MILCOM 2005. IEEE, pages 2642-2648 Vol. 4, Oct. 2005.

[4] B. Tavli and W. B. Heinzelman. MH-TRACE: Multi hop time reservation using adaptive control for energy efficiency. IEEE Journal on Selected Areas of Communications, 22(5):942-953, June 2004.

[5] T. Cooklev. Wireless Communication Standarts. IEEE Press, 2004.

[6] J. Karaoguz. High-rate wireless personal area networks. Communications Magazine, IEEE, 39(12):96-102, Dec 2001.

[7] T. Numanoglu, B. Tavli, and W. B. Heinzelman. The effects of channel errors on coordinated and non-coordinated medium access control protocols. In Proceedings of IEEE International Conference on Wireless and Mobile Computing, volume 1, pages 58-65, Aug 2005.

[8] V. H. MacDonald. Advanced mobile phone service: The cellular concept. Bell Syst. Tech. J., 58:15-41, 1979.

[9] D. J. Goodman, R. A. Valenzuela, K. T. Gayliard, and B. Ramamurthi. Packet reservation multiple access for local wireless communications. IEEE Trans. Commun., 37:885-890, Aug 1989.

[10] D. J. Goodman and S. X. Wei. Efficiency of packet reservation multiple access. IEEE Trans. Vehic. Tech., 40(1):170-176, Feb 1991.

[11] T. Camp, J. Boleng, and V. Davies. A survey of mobility models for ad hoc network research. Wireless Communications and Mobile Computing (WCMC), 2(5):483-502, 2002.

[12] C. Bettstetter and C. Wagner. The spatial node distribution of the random waypoint mobility model. In Mobile Ad-Hoc Netzwerke, WMAN 2002, pages 41-58. GI, 2002.

[13] W. B. Heinzelman, A. Chandrakasan, and H. Balakrishnan. An application-specific protocol architecture for wireless microsensor networks. IEEE Transactions on Wireless Communications, 1:660-670, 2002.

[14] Network simulator (ns-2). http://www.isi.edu/nsnam/ns. 